\title{
ON SOME CLASSES OF AC-OPERATORS
}

\author{
M. B. GHAEMI \\ Department of Mathematics, University of Birjand, Birjand, Iran \\ e-mail: Mohammadbg@yahoo.com
}

(Received 13 October, 2000; accepted 18 December 2000)

\begin{abstract}
AC-operators generalise normal operators on Hilbert space in the context of well-boundedness. In this paper we study AC-operators $T=U+i V$, where $U$ and $V$ are commuting well-bounded operators with decomposition of the identity of bounded variation. We also explore some properties of AC-operators by applying the theory of (Foiaş) decomposable operators.
\end{abstract}

2000 Mathematics Subject Classification. 47B15, 47B40.

1. Introduction. The class of well-bounded operators on a Banach space was introduced by Smart [14] as a natural analogue of selfadjoint operators on Hilbert space, and was first studied by Smart and Ringrose ([12], [13], [14]). Well-bounded operators are defined as those which possess a functional calculus for absolutely continuous functions on some compact interval $[a, b]$ of the real line. Smart and Ringrose [12] proved that on a reflexive Banach space a well-bounded operator can always be written as an integral with respect to a spectral family of projections. Ringrose showed that the dual of a well-bounded operator always admits an integral representation with respect to a family of projections. This family of projections was called a decomposition of the identity (a definition will be given in section 2). Wellbounded operators of type (B) were characterised by Berkson and Dowson [2] and by Spain [15] as being those for which the absolutely continuous functional calculus is weakly compact. Berkson and Dowson [2] introduced the class of well-bounded operators with decomposition of the identity of bounded variation. They showed that there exists a well-bounded operator with decomposition of the identity of bounded variation which is not "decomposable in $X$ " in that the projections in the decomposition of the identity are not the adjoints of projections on $X$.

In [4] Berkson and Gillespie introduced the concept of an AC-operator as an operator which possesses a functional calculus for the absolutely continuous functions on some rectangle in $\mathbb{C}$ (more detailed definitions will be given in section 3 ). Berkson and Gillespie showed that these operators can be characterised by the fact that they possess a splitting into real and imaginary parts, $T=U+i V$, where $U$ and $V$ are commuting well-bounded operators. They showed [4] that if $U$ and $V$ are wellbounded of type (B) this splitting is unique, and that if $S \in L(X)$ commutes with $U+i V$ then $S$ commutes with $U$ and $V$. Berkson, Doust and Gillespie later showed that neither result is guaranteed if the type (B) hypothesis is omitted [3].

The author would like to express sincere thanks to Dr P.G. Spain and Dr H.R. Dowson for encouragement and advice. Also the author is grateful to Professor T. A. Gillespie for his helpful comments. 
In Section 3 we study operators $U+i V$, where $U$ and $V$ are commuting wellbounded with decomposition of the identity of bounded variation. In this case also, if $S \in L(X)$ commutes with $U+i V$ then $S$ commutes with $U$ and $V$. It is shown if $T=$ $U+i V$, where $U$ and $V$ are commuting well-bounded operators with decomposition of the identity of bounded variation, and if either $X$ does not contain a copy of $c_{0}$, or if $U$ and $V$ are type (A) then such a representation is unique. An important tool is the fact that if $S^{\prime} \in L\left(X^{\prime}\right)$ is a scalar-type operator of class $X$ then $S \in L(X)$ is strongly normal-equivalent ([9, Theorem 2.4]). In Section 4, by applying the theory of (Foiaş) decomposable operators, we explore some properties of AC-operators $T=U+i V$ where $U, V$ are type (B) well-bounded operators or well-bounded operators with decomposition of the identity of bounded variation.

2. Well-bounded operators. Let $X$ be a complex Banach space with dual space $X^{\prime}$. The Banach algebra of all bounded linear operators on $X$ is denoted by $L(X)$. Given $T \in L(X)$, let $T^{\prime} \in L\left(X^{\prime}\right)$ be its adjoint, and let

$$
\|T\|=\sup \{\|T x\|: x \in X,\|x\| \leq 1\} .
$$

The theory of well-bounded operators is given in more detail in [8]. We shall give some of the basic definitions regarding well-bounded operators.

Definition 2.1. An operator $T \in L(X)$ is said to be well-bounded if there exist a constant $K$ and a closed interval $J=[a, b] \subseteq \mathbb{R}$ such that

$$
\|p(T)\| \leq K\left\{|p(a)|+\int_{a}^{b}\left|p^{\prime}(t)\right| d t\right\}
$$

for all complex polynomials $p$.

Definition 2.2. A decomposition of the identity for $X$ (on $J$ ) is a family

$$
\{E(s): s \in \mathbb{R}\}
$$

of projections on $X^{\prime}$ such that

(1) $E(s)=0$ for $s<a$ and $E(s)=I$ for $s \geq b$,

(2) $E(s) E(t)=E(t) E(s)=E(s)$ for $s \leq t$,

(3) there is a real constant $K$ such that $\|E(s)\| \leq K$ for $s \in \mathbb{R}$,

(4) the function $s \rightarrow\langle x, E(s) y\rangle$ is Lebesgue measurable for $x \in X$, and $y \in X^{\prime}$,

(5) for each $x \in X$, the map $y \mapsto \rightarrow\langle x, E(s) y\rangle$ from $X^{\prime}$ into $L^{\infty}[a, b]$ is continuous when $X^{\prime}$ and $L^{\infty}[a, b]$ are given their weak* topologies as the duals of $X$ and $L^{1}[a, b]$ respectively,

(6) if $x \in X, y \in X^{\prime}, s \in[a, b)$, and if the function $t \rightarrow \int_{a}^{t}\langle x, E(u) y\rangle d u$ is right differentiable at $s$, then the right derivative at $s$ is $\langle x, E(s) y\rangle$.

Given a decomposition of the identity $\{E(s): s \in \mathbb{R}\}$ there exists a unique wellbounded operator $T \in L(X)$ such that

$$
\langle T x, y\rangle=b\langle x, y\rangle-\int_{a}^{b}\langle x, E(u) y\rangle d u .
$$


Every well-bounded operator has such a representation, but in general the decomposition of the identity is not uniquely determined by $T$.

Definition 2.3. Let $T$ be a well-bounded operator and let $\{E(s): s \in \mathbb{R}\}$ be a decomposition of the identity for $T$. Then $T$ is well-bounded of type (A) if there exists a family of projections $F(s)$ on $X$ such that $F(s)^{\prime}=E(s)$ for all $s \in \mathbb{R}$.

This definition is equivalent to Definition 16.7 of [8], by ([5, Theorem 3.2]).

Definition 2.4. $T$ is said to be of type (B) if $T$ is of type (A) and, in addition, for each real $s, \lim _{t \rightarrow s^{-}} F(t) x$ exists for every $x \in X$.

Definition 2.5. Let $T \in L(X)$ be a well-bounded operator on $X$. A decomposition of the identity $\{E(s): s \in \mathbb{R}\}$ for $T$ is said to be of bounded variation if the function $s \mapsto\left\langle x, E(s) y^{\prime}\right\rangle$ is of bounded variation on $\mathbb{R}$ for every $x \in X$ and $y^{\prime} \in X^{\prime}$.

Let $T \in L\left(\ell^{2}\right)$ denote the well-bounded operator constructed in ([10, Lemma 1]). $T$ is a well-bounded operator of type (B) which is not a well-bounded operator with decomposition of the identity of bounded variation. ([8, Example 16.19]) shows that there exists a well-bounded operator of type (A) with decomposition of the identity of bounded variation which is not well-bounded of type (B). This shows that neither of the classes of well-bounded operators with decomposition of the identity of bounded variation and of well-bounded operators of type (B) includes the other.

Definition 2.6. An operator $T \in L(X)$ is hermitian if

$$
\|\exp (i t T)\|=1 \quad(t \in \mathbb{R}) .
$$

Definition 2.7. An operator $R \in L(X)$ is hermitian-equivalent if and only if there exists an equivalent norm on $X$ with respect to which $R$ is hermitian.

Equivalently, $R$ is hermitian-equivalent if and only if there is an $M(\geq 1)$ such that

$$
\|\exp (i t R)\| \leq M \quad(t \in \mathbb{R}) .
$$

If this condition is satisfied, then

$$
|x|=\sup \{\|\exp (i t R) x\|: t \in \mathbb{R}\}
$$

defines a norm on $X$, equivalent to $\|\cdot\|$, with respect to which $R$ is hermitian.

More generally, a set $\Lambda \subseteq L(X)$ is hermitian-equivalent if and only if there is an equivalent norm on $X$ with respect to which every operator in $\Lambda$ is hermitian. It is known ([8, Theorem 4.17]) that when $\Lambda$ is a commutative subset of $L(X)$, then $\Lambda$ is hermitian-equivalent if and only if each operator in the closed real linear span of $\Lambda$ is hermitian-equivalent.

Definition 2.8. The operator $T=R+i J$ is normal-equivalent if $R J=J R$ and the set $\{R, J\}$ is hermitian-equivalent.

If $T \in L(X)$ is normal-equivalent then $T$ can be expressed uniquely in the form $R+i J$, where $R J=J R$ and the set $\{R, J\}$ is hermitian-equivalent ([9, Lemma 1.10]).

THEOREM 2.9. If $T$ is a well-bounded operator with decomposition of the identity of bounded variation then the set $\left\{T^{n}: n=0,1,2, \cdots\right\}$ is hermitian-equivalent. 
Proof. If $T$ is a well-bounded operator with decomposition of the identity of bounded variation, then, by [8, Theorem 16.15], $T^{\prime}$ is real scalar-type of class $X$ with resolution of the identity $E(\cdot)$. Then by [9, Theorem 2.5] there are operators $H, K \in L(X)$ such that $\left\{H^{n} K^{m}: n, m=0,1,2, \cdots\right\}$ is hermitian-equivalent and $H^{\prime}=\int_{\sigma\left(T^{\prime}\right)} \operatorname{Re} \lambda E(d \lambda)$ and $K^{\prime}=\int_{\sigma\left(T^{\prime}\right)} \operatorname{Im} \lambda E(d \lambda)$. Note that $\sigma(T) \subseteq \mathbb{R}$ so that $\sigma\left(T^{\prime}\right)$ $\subseteq \mathbb{R}:$ then $K^{\prime}=0$ : so that $T=H$.

Definition 2.10. An operator $T \in L(X)$ is said to have the single-valued extension property if whenever $f: D_{f} \rightarrow X$ is analytic in an open set $D_{f} \subseteq \mathbb{C}$ and satisfies

$$
(\lambda I-T) f(\lambda)=0, \quad\left(\lambda \in D_{f}\right)
$$

it follows that $f=0$ in $D_{f}$.

Let $T \in L(X)$ have the single-valued extension property. Given $x \in X$ we denote by $\varrho_{T}(x)$ the set of elements $\alpha \in \mathbb{C}$ such that there exists an $X$-valued function $x(\lambda)$ analytic in a neighbourhood $V_{\alpha}$ of $\alpha$, such that

$$
(\lambda I-T) x(\lambda)=x \quad\left(\lambda \in V_{\alpha}\right) .
$$

In particular, $\varrho(T) \subseteq \varrho_{T}(x)$. The complement $\sigma_{T}(x)=\mathbb{C} \backslash \varrho_{T}(x)$ is the local spectrum of $T$ at $x$; it is a compact subset of $\sigma(T)$, and is non-empty for $x \neq 0$.

For $F \subseteq \mathbb{C}$, let $X_{T}(F)=\left\{x \in X: \sigma_{T}(x) \subseteq F\right\}$. This is a $T$-invariant manifold.

Lemma 2.11. Let $U$ and $U_{1}$ be commuting well-bounded operators on $X$, where $U$ is well-bounded of type (A) and $U-U_{1}$ is quasinilpotent. Then $U=U_{1}$.

Proof. Let $\{E(s): s \in \mathbb{R}\}$ be the family of projections on $X$ whose adjoints form a decomposition of the identity of $U$. By [8, Theorem 15.19 (iii) and 16.3] there exist a decomposition of the identity $\{F(s): s \in \mathbb{R}\}$ for $U_{1}$ such that $E(s)^{\prime} F(s)=$ $F(s) E(s)^{\prime}(s \in \mathbb{R})$. Observe that $U^{\prime}$ and $U_{1}^{\prime}$ have the single valued extension property ([8, Proposition 5.28]). Given $x^{\prime} \in X^{\prime}$ denote by $\sigma_{U_{1}}\left(x^{\prime}\right)$ the local spectrum of $x^{\prime}$ relative to $U_{1}{ }^{\prime}$. Since $U-U_{1}$ is quasinilpotent, so also is $U^{\prime}-U_{1}^{\prime}$. Hence $\sigma_{U_{1}}\left(x^{\prime}\right)$ is equal to the local spectrum $x^{\prime}$ relative to $U^{\prime}$ by [6, Theorem 1.2.4]. Hence by ([2, Theorem 5.6]) the projections $E(s)^{\prime}$ and $F(s)$ have the same range, and hence are equal, since they commute. This is true for all $s \in \mathbb{R}$, and so $U=U_{1}$.

COROLlaRY 2.12. If $U$ is a quasinilpotent well-bounded operator, then $U=0$.

3. AC-operators One of the major complications one encounters when trying to extend this theory to operators with complex spectra is deciding upon the correct concept of an absolutely continuous function of two variables to use. In the discussion that follows we shall identify the subsets of $\mathbb{R}^{2}$ with subsets of $\mathbb{C}$ in the usual way. Let $m$ denote Lebesgue measure on $\mathbb{R}^{2}$. Let $J=[a, b]$ and $K=[c, d]$ be two compact intervals in $\mathbb{R}$. Let $\Lambda$ be a rectangular partition of $J \times K$ :

$$
a=s_{0}<s_{1}<\cdots<s_{n}=b, \quad c=t_{0}<t_{1}<\cdots<t_{m}=d .
$$


For a function $f: J \times K \rightarrow \mathbb{C}$, define

$$
V_{\Lambda}(f)=\sum_{i=1}^{n} \sum_{j=1}^{m}\left|f\left(s_{i}, t_{j}\right)-f\left(s_{i}, t_{j-1}\right)-f\left(s_{i-1}, t_{j}\right)+f\left(s_{i-1}, t_{j-1}\right)\right| .
$$

The variation of $f$ is defined to be

$$
\operatorname{var}_{J \times K}(f)=\sup \left\{V_{\Lambda}(f): \Lambda \text { is a rectangular partition of } J \times K\right\} .
$$

We shall say that the function $f$ is of bounded variation if $\operatorname{var}_{J \times K} f, \operatorname{var}_{J} f(\cdot, d)$ and $\operatorname{var}_{K} f(b, \cdot)$ are all finite. The set $B V(J \times K)$ of all functions $f: J \times K \rightarrow \mathbb{C}$ of bounded variation is a Banach algebra under the norm

$$
\left\||f \||=|f(b, d)|+\operatorname{var}_{J} f(\cdot, d)+\operatorname{var}_{K} f(b, \cdot)+\operatorname{var}_{J \times K} f .\right.
$$

A function $f: J \times K \rightarrow \mathbb{C}$ is said to be absolutely continuous if

(1) for all $\epsilon>0$, there exists $\delta>0$ such that

$$
\sum_{R \in \Re} \operatorname{var}_{R} f<\epsilon
$$

whenever $\mathfrak{R}$ is a finite collection of non-overlapping subrectangles of $J \times K$ with $\sum_{R \in \Re} m(R)<\delta$;

(2) The marginal functions $f(\cdot, d)$ and $f(b, \cdot)$ are absolutely continuous functions on $J$ and $K$ respectively.

The set $\mathrm{AC}(J \times K)$ of all absolutely continuous functions $f: J \times K \rightarrow \mathbb{C}$ is a Banach subalgebra of $\mathrm{BV}(J \times K)$, and is the closure in $\mathrm{BV}(J \times K)$ of the polynomials in two real variables on $J \times K$. Equivalently, one can consider $\mathrm{AC}(J \times K)$ to be the closure of the polynomial functions $p(z, \bar{z})$ on $J \times K \subseteq \mathbb{C}$.

Define the functions $u, v \in \operatorname{AC}(J \times K)$ by $u(x, y)=x$ and $v(x, y)=y$.

Definition 3.1. An operator $T \in L(X)$ is said to be an AC-operator if there exists a continuous unital Banach algebra homomorphism $\Theta: \mathrm{AC}(J \times K) \rightarrow L(X)$ for which $\Theta(u+i v)=T$.

Berkson and Gillespie ([4, Theorem 5]) proved that this is equivalent to the condition that $T$ can be written as $T=U+i V$ where $U$ and $V$ are commuting wellbounded operators on $X$. They showed that if $U$ and $V$ are well-bounded of type (B) the representation in the form $T=U+i V$ is unique and if $S \in L(X)$ commutes with $T$ then $S$ commutes with $U$ and $V$. We generalise this result in section 4 . However, neither result is guaranteed if the type (B) hypothesis is omitted as is shown by [3, Examples 3.1 and 3.4].

THeOREM 3.2. Let $U$ and $V$ be commuting well-bounded operators with decompositions of the identity of bounded variation on $X$ and let $S \in L(X)$ commute with $U+i V$. Then $S$ commutes with $U$ and $V$.

Proof. By Theorem 2.9 the operators $U, V$ are hermitian-equivalent. Since $U V=V U$ it follows that $U+i V$ is normal-equivalent. By [8, Theorem 4.22] we have $S U=U S, S V=V S$. 
By applying the theory of (Foiaş) decomposable operators we generalise Theorem 3.2 in Section 4.

TheOREM 3.3. Let

$$
T=U+i V=U_{1}+i V_{1},
$$

where

(1) $U$ and $V$ are commuting well-bounded operators of type (A) with decompositions of the identity of bounded variation;

(2) $U_{1}$ and $V_{1}$ are commuting well-bounded operators on $X$.

Then $U=U_{1}$ and $V=V_{1}$.

Proof. $U_{1}$ commutes with $U_{1}+i V_{1}$ and hence $U_{1}$ commutes with $U+i V$. By Theorem 3.2, $U_{1}$ commutes with $U$ and $V$. Similarly $V_{1}$ commutes with $U$ and $V$. Hence the set $\left\{U, V, U_{1}, V_{1}\right\}$ is commutative. Since well-bounded operators have real spectra, we can apply standard Gelfand theory to deduce that $U-U_{1}$ and $V-V_{1}$ are quasinilpotent. Now, by Lemma 2.11, $U=U_{1}$ and $V=V_{1}$.

When $X$ does not contain a copy of $c_{0}$ we need not assume that the real and imaginary parts are decomposable in $X$.

Theorem 3.4. Suppose that $X$ does not contain a copy of $c_{0}$. Let

$$
T=U+i V=U_{1}+i V_{1},
$$

where

(1) $U$ and $V$ are commuting well-bounded operators on $X$ with decomposition of the identity of bounded variation,

(2) $U_{1}$ and $V_{1}$ are commuting well-bounded operators on $X$.

Then $U=U_{1}$ and $V=V_{1}$.

Proof. $U$ and $V$ are real scalar-type spectral operators ([7, Theorem 2]). Now by [8, Theorem 16.17] $U$ and $V$ are type (A) well-bounded operators. The result follows from Theorem 3.3.

\section{AC-operators and (Foiaş) decomposable operators.}

Definition 4.1. Let $X$ be a Banach space and $T \in L(X)$. A closed linear subspace $\mathcal{Y}$ of $X$ is called a spectral maximal space of $T$ if

(1) $\mathcal{Y}$ is invariant under $T$,

(2) if $\mathcal{Z}$ is another closed linear subspace of $X$, invariant under $T$, such that $\sigma(T \mid \mathcal{Z}) \subseteq \sigma(T \mid \mathcal{Y})$, then $\mathcal{Z} \subseteq \mathcal{Y}$.

A spectral maximal space of $T \in L(X)$ is ultra-invariant under $T$; that is it is invariant under any operator commuting with $T$.

Definition 4.2. An operator $T \in L(X)$ is called decomposable if any open cover $\mathbb{C}=G_{1} \cup G_{2}$ of the complex plane $\mathbb{C}$ by two sets $G_{1}$ and $G_{2}$ yields a splitting of the 
spectrum $\sigma(T)$ and of the space $X$ in the sense that there exist closed $T$-invariant linear subspaces $Y$ and $Z$ of $X$ for which $\sigma(T \mid Y) \subseteq G_{1}, \sigma(T \mid Y) \subseteq G_{2}$ and $X=Y+Z$.

The definition of decomposability has been simplified considerably since Colojoară and Foiaş wrote their book [6]. The original definition of a decomposable operator as developed by Foiaş was somewhat complicated and involved the notion of a spectral maximal space. See [11], [6] and [16] for an account of the classical theory of decomposable operators.

Let $\Omega$ be a subset of the complex plane. An algebra $\mathcal{A}$ of complex functions defined on $\Omega$ is called normal if for every open finite covering $\left\{G_{i}\right\}_{1 \leq i \leq n}$ of $\bar{\Omega}$ there exist functions $f_{i} \in \mathcal{A}$ such that

(1) $f_{i}(\Omega) \subseteq[0,1], \quad(1 \leq i \leq n)$,

(2) $\operatorname{supp}\left(f_{i}\right) \subseteq G_{i}, \quad(1 \leq i \leq n) \quad$ where $\operatorname{supp}\left(f_{i}\right)=\overline{\{\lambda \in \Omega \mid f(\lambda \neq 0)\}}$

(4) $\sum_{i=1}^{n} f_{i}=1 \quad$ on $\Omega$.

Definition 4.3. Let $\mathcal{A}$ be an algebra of complex functions defined on the closed set $\Omega \subseteq \mathbb{C}$. $\mathcal{A}$ will be called topologically admissible if

(1) $\lambda \in \mathcal{A}, 1 \in \mathcal{A}$ (where $\lambda$ is the function $f(\lambda)=\lambda$, and 1 is the function $f(\lambda)=1)$,

(2) $\mathcal{A}$ is normal,

(3) $\mathcal{A}$ is endowed with a locally convex topology $\tau$ such that if $\left\{f_{n}\right\} \subseteq \mathcal{A}$ is a Cauchy sequence in $\tau$ and $f_{n}(\lambda) \rightarrow 0$ for every $\lambda \in \Omega$, then $f_{n} \rightarrow 0$ in $\tau$,

(4) for every $f \in \mathcal{A}$ and every $\xi \notin \operatorname{supp}(f)$, the function

$$
f_{\xi}(\lambda)= \begin{cases}\frac{f(\lambda)}{\xi-\lambda} & \text { if } \lambda \in \Omega \backslash\{\xi\}, \\ 0 & \text { if } \lambda \in \Omega \cap\{\xi\}\end{cases}
$$

belongs to $\mathcal{A}$, and the mapping $\xi \rightarrow f_{\xi}$ of $\mathbb{C} \backslash \operatorname{supp}(f)$ into $\mathcal{A}$ is continuous.

Lemma 4.4. Let $J=[a, b], K=[c, d]$, and $\Omega=J \times K$; let $\mathcal{A}=\operatorname{AC}(\Omega)$ and $f \in \mathcal{A}$ and let $f_{\xi}$ be as in Definition 4.3. Then

(1) $f_{\xi} \in \mathrm{AC}(J \times K)$,

(2) the mapping $\xi \rightarrow f_{\xi}$ of $\mathbb{C} \backslash \operatorname{supp}(f)$ into $\mathcal{A}$ is continuous.

Proof. Let $\xi \notin \operatorname{supp}(f)$. We can find a $C^{\infty}$ function $\kappa_{f, \xi}$ and a closed disc $D_{\xi} \quad$ containing $\xi$ such that $\left.\kappa_{f, \xi}\right|_{\operatorname{supp}(f)}=1$ and $\left.\kappa_{f, \xi}\right|_{D_{\xi}}=0$; then $\lambda_{1} \rightarrow \frac{\kappa_{f, \xi}}{\xi-\lambda} \in C^{\infty}$ and $f_{\xi}(\lambda)=\frac{f(\lambda) \kappa_{f, \xi}(\lambda)}{\xi-\lambda}$, so is in $\operatorname{AC}(J \times K)$.

The mapping $\xi \rightarrow f_{\xi}$ is clearly continuous.

Corollary 4.5. $\mathrm{AC}(J)$ and $\mathrm{AC}(J \times K)$ are topologically admissible algebras.

Definition 4.6 Let $\mathcal{A}$ be a topologically admissible algebra. A mapping $\Theta: \mathcal{A} \rightarrow L(X)$ is called a continuous $\mathcal{A}$-spectral function if

(1) $\Theta: \mathcal{A} \rightarrow L(X)$ is an algebraic homomorphism, and $\Theta(1)=I$,

(2) $\Theta: \mathcal{A} \rightarrow L(X)$ is continuous ([6, Definition 3.5.3]). 
Remark 4.7. By [6, Theorem 3.5.4] every continuous $\mathcal{A}$-spectral function is an $\mathcal{A}$-spectral function in the sense of [6, Definition 3.1.3].

Definition 4.8. An operator $S \in L(X)$ is called $\mathcal{A}$-scalar if there exists an $\mathcal{A}$-spectral function $\Theta$ such as $\Theta(\lambda)=S$. Such an $\mathcal{A}$-spectral function will be called an $\mathcal{A}$-spectral function of $S$.

By Corollary 4.5 well-bounded operators are $\mathcal{A}$-scalar and hence by [6, Theorem 3.1.16] they are decomposable operators. If $T \in L(X)$ is a decomposable operator then for any closed subset $F$ of $\sigma(T)$ the subspace $X_{T}(F)$ is a spectral maximal space of $T$ [6, Theorem 2.1.5].

Theorem 4.9. Let $T \in L(X)$ be a well-bounded operator, $x_{0} \in X$ and $\lim _{n \rightarrow \infty}\left\|T^{n} x_{0}\right\|^{1 / n}=0$. Then $T x_{0}=0$.

Proof. $T$ is decomposable. Hence $X_{T}(\{0\})$ is a closed subspace of $X$ which is invariant for $T$ and satisfies $\sigma\left(T \mid X_{T}(\{0\})\right)=\{0\} \cap \sigma(T)$ ([6, Theorem 2.1.5]). Therefore, $T \mid X_{T}(\{0\})$ is a quasinilpotent well-bounded operator. Hence, by Corollary 2.12, $T \mid X_{T}\left(\{0\}=0\right.$. Now by $\left[\mathbf{6}\right.$, Lemma 4.4.4] we have $X_{T}(\{0\})=\left\{x \in X: \lim _{n \rightarrow \infty}\right.$ $\left.\left\|T^{n} x\right\|^{1 / n}=0\right\}$. Thus $x_{0} \in X_{T}(\{0\})$ and $T x_{0}=0$.

Corollary 4.10. Let $T=U+i V$ where $U, V$ are commuting well-bounded operators. If $\lim _{n \rightarrow \infty}\left\|T^{n} x\right\|^{1 / n}=0$ for some $x \in X$, then $U x=V x=0$.

Proof. There is a continuous homomorphism $\Theta: \mathrm{AC}(J \times K) \rightarrow L(X)$ such that $\Theta(u)=U, \Theta(v)=V$ and $\Theta(u+i v)=U+i V$.

$$
\lim _{n \rightarrow \infty}\left\|T^{n} x\right\|^{1 / n}=0
$$

Hence $x \in X_{T}(\{0\})([6$, Lemma 4.4.4]). This gives

$$
x \in X_{T}(\{0\}) \subseteq X_{T}\left(u^{-1}(\{0\})=X_{\Theta(u)}(\{0\})=X_{U}(\{0\}),\right.
$$

where the inclusion is by [6, Theorem 1.1.2] and the first equality is by [6, Theorem 3.2.4]. Hence

$$
\lim _{n \rightarrow \infty}\left\|U^{n} x\right\|^{1 / n}=0
$$

By Theorem 4.9, $U x=0$. Similarly we can show that $V x=0$.

Lemma 4.11 is due to Gillespie (private communication).

Lemma 4.11. Let $T=U+i V$, where $U, V$ are commuting type (B) well-bounded operators on a Banach space $X$. Fix $a, b \in \mathbb{R}$ with $a<b$ and let $F=\{z \in \mathbb{C}: a \leq$ $\operatorname{Re} z \leq b\}$. Then

$$
X_{T}(F)=\left[E(b)-E\left(a^{-}\right)\right] X
$$

where $E(\cdot)$ is the spectral family of $U$. 
Proof. Standard Gelfand theory shows that the spectrum of the restriction of $T$ to $\left[E(b)-E\left(a^{-}\right)\right] X$ is contained in $F$. Hence $\left[E(b)-E\left(a^{-}\right)\right] X \subseteq X_{T}(F)$. Now $U$ and $V$ commute with $T$, and hence $X_{T}(F)$ is invariant under both $U$ and $V$ ([6, Theorem 2.3.3]). Again, standard Gelfand theory implies that the spectrum of the restriction of $U$ to $X_{T}(F)$ is contained in $[a, b]$. It now follows from [8, Theorem 19.3] that

$$
X_{T}(F) \subseteq\left[E(b)-E\left(a^{-}\right)\right] X .
$$

Let $X$ and $Y$ be two Banach spaces. $L(X, Y)$ will denote the collection of all bounded linear mappings of $X$ into $Y$. For $T \in L(X)$ and $S \in L(Y)$ we define

$$
L(S), R(T), C(S, T): L(X, Y) \rightarrow L(X, Y)
$$

by $L(S) A=S A, R(T) A=A T$ (where $A \in L(X, Y)$ ) and $C(T, S)=L(S)-R(T)$, respectively.

We can now prove the following theorem, which generalises that of Berkson and Gillespie ([4, Lemma 4]).

Theorem 4.12. Suppose $X$ and $Y$ are Banach spaces. Let $T_{1}=U_{1}+i V_{1} \in L(X)$ and $T_{2}=U_{2}+i V_{2} \in L(Y)$ be AC-operators where $U_{i}, V_{i},(i=1,2)$ are type $(\mathrm{B})$ wellbounded operators. Let $S \in L(X, Y)$ be an operator such that

$$
\lim _{n \rightarrow \infty}\left\|C\left(T_{2}, T_{1}\right)^{n} S\right\|^{1 / n}=0 .
$$

Then $U_{2} S=S U_{1}, V_{2} S=S V_{1}$ and $T_{2} S=S T_{1}$.

Proof. It is sufficient to show that $E_{2}(a) S=S E_{1}(a),(a \in \mathbb{R})$, where $\left\{E_{1}(s)\right.$ : $s \in \mathbb{R}\}$ and $\left\{E_{2}(s): s \in \mathbb{R}\right\}$ are the spectral families of $U_{1}$ and $U_{2}$ respectively. Let $a, b \in \mathbb{R}, a<b$ and $F=\{z \in \mathbb{C}: a \leq \operatorname{Re} z \leq b\}$. By [6, Theorem 2.3.3] we have $S X_{T_{1}}(F) \subseteq Y_{T_{2}}(F)$ and hence $S\left[E_{1}(b)-E_{1}\left(a^{-}\right)\right] X \subseteq\left[E_{2}(b)-E_{2}\left(a^{-}\right)\right] Y$ (Lemma 4.11). If $\left\{a_{n}\right\}$ is a sequence decreasing to $a$, then $E_{i}\left(a_{n}\right) \rightarrow E_{i}(a)(i=1,2)$ strongly and $S\left[E_{1}(b)-E_{1}\left(a_{n}{ }^{-}\right)\right] X \subseteq\left[E_{2}(b)-E_{2}\left(a_{n}{ }^{-}\right)\right] Y$ then gives that

$$
S\left[E_{1}(b)-E_{1}(a)\right] X \subseteq\left[E_{2}(b)-E_{2}(a)\right] Y .
$$

Taking $b$ sufficiently large and positive we get $S\left[I-E_{1}(a)\right] X \subseteq\left[I-E_{2}(a)\right] Y$ and taking $a$ sufficiently large and negative we get that $S E_{1}(b) X \subseteq E_{2}(b) Y$. Hence for $a \in \mathbb{R}$ we have $S E_{1}(a) X \subseteq E_{2}(a) Y, S\left[I-E_{1}(a)\right] X \subseteq\left[I-E_{2}(a)\right] Y$ and therefore $E_{2}(a) S=S E_{1}(a)$.

Recall that two operators $T, S \in L(X)$ are said to be quasinilpotent equivalent, $T \stackrel{q}{\sim} S$ ([6, Definition 1.21]), if and only if

$$
\lim _{n \rightarrow \infty}\left\|C(T, S)^{n} I\right\|^{1 / n}=0=\lim _{n \rightarrow \infty}\left\|C(S, T)^{n} I\right\|^{1 / n}=0 .
$$

Corollary 4.13. Suppose $X$ and $Y$ are Banach spaces. Let $T_{1}=U_{1}+i V_{1} \in L(X)$ and $T_{2}=U_{2}+i V_{2} \in L(X)$ be AC-operators where $U_{i}, V_{i},(i=1,2)$ are type (B) wellbounded operators. Suppose $T_{1} \stackrel{q}{\sim} T_{2}$. Then $U_{1}=U_{2}, \quad V_{1}=V_{2}$ and $T_{1}=T_{2}$. 
Proof. With $Y=X$ and $S=I$ in Theorem 4.12 we obtain $U_{1}=U_{2}, V_{1}=V_{2}$ and $T_{1}=T_{2}$.

The following results are immediate corollaries of Theorem 2.9 and [1, Theorem 1].

Corollary 4.14. Suppose $X$ and $Y$ are Banach spaces. Let $T_{1}=U_{1}+i V_{1} \in L(X)$ and $T_{2}=U_{2}+i V_{2} \in L(Y)$ be AC-operators, where $U_{i}, V_{i}(i=1,2)$ are commuting well-bounded operators with decompositions of the identity of bounded variation on $X, Y$. Suppose further that

$$
\lim _{n \rightarrow \infty}\left\|C\left(T_{2}, T_{1}\right)^{n} S\right\|^{1 / n}=0 .
$$

Then $U_{2} S=S U_{2}, V_{2} S=S V_{1}$, and $T_{2} S=S T_{1}$.

Corollary 4.15. Let $X$ be a Banach space and let $T_{i}=U_{1}+i V_{1} \in L(X)$ $(i=1,2)$ be AC-operators with $U_{i}, V_{i},(i=1,2)$ commuting well-bounded operators with decompositions of the identity of bounded variation. Suppose $T_{1} \stackrel{q}{\sim} T_{2}$. Then $U_{1}=U_{2}, \quad V_{1}=V_{2}$ and $T_{1}=T_{2}$.

\section{REFERENCES}

1. E. Albrecht, On some classes of generalized spectral operators. Arch. Math. (Basel) 30 (1978), 297-303.

2. E. Berkson and H.R. Dowson, On uniquely decomposable well-bounded operators, Proc. London Math Soc. (3), 22 (1971), 339-358.

3. E. Berkson, I. Doust and T. A. Gillespie, Properties of AC-operators, Acta Sci. Math. (Szeged), 63 (1997), 249-257.

4. E. Berkson and T. A. Gillespie, Absolutely continuous functions of two variables and well-bounded operators, J. London Math. Soc (2), 30 (1984), 305-321.

5. Cheng Qingping and I. Doust, Some remarks on well-bounded and scalar-type decomposable operators, (to appear).

6. I. Colojoară and C. Foiaş, Theory of generalized spectral operators (Gordon and Breach, New York, 1968).

7. I, Doust, Well-bounded and scalar-type spectral operators on spaces not containing $c_{0}$, Proc. Amer. Math. Soc. 105 (1989), 367-370.

8. H. R. Dowson, Spectral theory of linear operators (Academic Press, 1978).

9. M. B. Ghaemi, Normal-equivalent operators and operators with dual of scalar-type, Proc. Edinburgh Math. Soc. 43 (2000), 261-268.

10. T. A. Gillespie, Commuting well-bounded operators on Hilbert spaces, Proc. Edinburgh Math. Soc. (2), 20 (1976), 167-172.

11. K. B. Laursen and M. N. Neumann, An introduction to local spectral theory (Clarendon Press, Oxford, 2000). $334-343$

12. J. R. Ringrose, On well-bounded operators, J. Austral. Math. Soc. Ser. A, 1 (1960),

13. J. R. Ringrose, On well-bounded operators II, Proc. London Math. Soc. (3), 13 (1963), 613-638.

14. D. R. Smart, Conditionally convergent spectral expansions, J. Austral. Math. Soc. Ser. A, 1 (1960), 319-333.

15. P. G. Spain, On well-bounded operators of type (B), Proc. Edinburgh Math. Soc. (2), 18 (1972), 35-48.

16. F.-H. Vasilescu, Analytic functional calculus and spectral decomposition, (D. Reidel Publ. Co. and Editura Acad. Rep. Social. Romania, Bucharest, 1982). 